\title{
Logging in: a comparative analysis of electronic health records versus anesthesia resident-driven logbooks
}

\section{L'enregistrement : une analyse comparative des dossiers médicaux informatisés par rapport aux logbooks des résidents en anesthésie}

\author{
Ryan McGinn, MD, MSc • Alexander J. Lingley, MSc • Daniel I. McIsaac, MD, MPH • \\ Christopher Pysyk, MD • Meghan C. McConnell, PhD • Gregory L. Bryson, MD • \\ Daniel Dubois, MD
}

Received: 10 January 2020/Revised: 12 May 2020/ Accepted: 15 May 2020/Published online: 13 July 2020

(C) Canadian Anesthesiologists' Society 2020

\begin{abstract}
Purpose Resident logbooks (RLBs) documenting clinical case exposure are widespread in medical education despite evidence of poor accuracy. Electronic health records (e.g., anesthesia information management systems [AIMS]) may provide advantages for auditing longitudinal case exposure. We evaluated the agreement between AIMS and RLBs for tracking case exposure during anesthesiology residency.

Methods We performed a historical cohort study with anesthesiology residents (2011-2018, all of whom used a RLB contemporaneously with AIMS) working in a multisite academic health sciences network. The primary outcome
\end{abstract}

R. McGinn, MD, MSc · C. Pysyk, MD

Department of Anesthesiology and Pain Medicine, The Ottawa

Hospital, University of Ottawa, Ottawa, ON, Canada

\section{A. J. Lingley, MSc}

Faculty of Medicine, University of Ottawa, Ottawa, ON, Canada

D. I. McIsaac, MD, MPH

Department of Anesthesiology and Pain Medicine, The Ottawa

Hospital, University of Ottawa, Ottawa, ON, Canada

Ottawa Hospital Research Institute, University of Ottawa,

Ottawa, ON, Canada

School of Epidemiology and Public Health, University of Ottawa, Ottawa, ON, Canada

M. C. McConnell, $\mathrm{PhD}$

Department of Innovation in Medical Education, University of Ottawa, Ottawa, ON, Canada was total case-load logging; secondary outcomes were volumes for seven surgical specialties (general, gynecology, neuro, orthopedic, thoracic, urology, and vascular surgery). Correlation of case numbers tracked by AIMS vs RLB was assessed using Pearson correlation; agreement was determined using Bland-Altman plots and intraclass correlation coefficients (ICC).

Results Data from 27 anesthesiology residents were collected. Overall, mean (standard deviation) case numbers were generally greater with AIMS vs RLB [649 (103) vs 583 (191); $P=0.049$ ). Total case volumes between systems had moderate correlation $(r=0.50)$ and agreement (intraclass correlation coefficient [ICC], 0.42;

G. L. Bryson, MD

Department of Anesthesiology and Pain Medicine, The Ottawa Hospital, University of Ottawa, Ottawa, ON, Canada

Ottawa Hospital Research Institute, University of Ottawa, Ottawa, ON, Canada

D. Dubois, MD $(\bowtie)$

Department of Anesthesiology and Pain Medicine, The Ottawa Hospital, University of Ottawa, Ottawa, ON, Canada e-mail: dadubois@toh.ca

Ottawa Hospital Research Institute, University of Ottawa, Ottawa, ON, Canada

Department of Innovation in Medical Education, University of Ottawa, Ottawa, ON, Canada

Department of Anesthesiology and Pain Medicine, The Ottawa Hospital, 1053 Carling Ave, Room B311, Ottawa, ON, Canada 
95\% CI, 0.34 to 0.59). Bland-Altman plots showed variable agreement between AIMS and RLB data [mean (SD) bias $=66$ (166) cases]. For general, gynecology, neuro, orthopedic, thoracic, urology, and vascular surgery, there was a range of poor to moderate agreement (ICC, 0.23-0.57) between AIMS and RLB.

Conclusion For anesthesiology resident case-logging, the number of cases logged in an AIMS was higher with lower variance compared with RLBs. Anesthesia information management systems vs RLB data showed low-moderate correlation and agreement. Given the additional time and resources required for RLBs, AIMS may be a superior method for tracking cases where available.

\section{Résumé}

Objectif Les logbooks des résidents documentant leur exposition à des cas cliniques sont répandus dans la formation médicale et ce, malgré des données probantes déplorant leur manque de précision. Les dossiers médicaux informatisés (par ex., les systèmes de gestion de l'information en anesthésie [SGIA]) pourraient présenter des avantages pour le contrôle de l'exposition longitudinale des résidents aux cas. Notre étude a évalué la concordance entre les SGIA et les logbooks pour l'exposition aux cas pendant la résidence en anesthésiologie.

Méthode Nous avons réalisé une étude de cohorte historique auprès de résidents en anesthésiologie (20112018, tous les résidents ayant utilisé un logbook simultanément aux SGIA) travaillant dans un réseau universitaire de sciences de la santé multisite. Le critère d'évaluation principal était l'enregistrement du nombre de cas total; les critères d'évaluation secondaires comprenaient les volumes pour sept spécialités chirurgicales (soit la chirurgie générale, gynécologique, orthopédique, thoracique, urologique, vasculaire et la neurochirurgie). La corrélation entre le nombre de cas enregistrés dans les SGIA vs les logbooks a été évaluée à l'aide d'une corrélation de Pearson; l'agrément a été déterminé à l'aide d'un graphique de Bland-Altman et de coefficients de correlation intraclasse (CCI).

Résultats Les données de 27 résidents en anesthésiologie ont été colligées. Globalement, les nombres de cas moyens (écart type) étaient en général plus élevés dans les SGIA que dans les logbooks [649 (103) vs 583 (191); P =0,049). Les volumes de cas totaux entre les systèmes présentaient une corrélation $(r=0,50)$ et un agrément (coefficient de correlation intraclasse [CCI], 0,42; IC $95 \%, 0,34$ à 0,59) modérés. Les graphiques de Bland-Altman ont démontré un agrément variable entre les données des SGIA et celles des logbooks [biais moyen $(E ́ T)=66$ (166) cas]. Pour les chirurgies générales, gynécologiques, orthopédiques, thoraciques, urologique, vasculaires et neurochirurgies, l'agrément allait de faible à modéré (CCI, 0,23-0,57) entre les SGIA et les logbooks.

Conclusion En ce qui a trait à l'enregistrement des cas des résidents en anesthésiologie, le nombre de cas enregistrés dans un SGIA était plus élevé et présentait une variance moindre que dans les logbooks. Les données des systèmes de gestion de l'information en anesthésie vs des logbooks ont affiché une correlation et un agrément faible à modéré. Étant donné le temps et les ressources supplémentaires nécessaires pour compléter les logbooks, les SGIA pourraient constituer une méthode supérieure pour le suivi des cas, lorsqu'un tel système est disponible.

\section{Keywords}

Logbook, competency-based medical education . clinical experience $\cdot$ anesthesiology

\section{Introduction}

The transition from a time-based learning model to competency-based medical education has emphasized the importance of robust, multi-faceted trainee assessment. ${ }^{1,2}$ Evaluation during competency-based medical education necessitates that residents show predefined competencies to provide safe and effective patient care. Self-logging of clinical encounters has been strongly encouraged by the Royal College of Physicians and Surgeons of Canada to document the acquisition of sufficient clinical exposure to achieve competency. ${ }^{3,4}$ Since 2004, Canadian anesthesiology residency programs have employed selfreported electronic logbooks (resident log books [RLBs]) that record case volume during postgraduate training. Such RLBs can identify gaps in clinical exposure that may reflect deficiencies in training. Once identified, these gaps can direct residents to seek additional clinical experience. $^{5,6}$ Logbooks are also used by program directors and accreditation bodies to document sufficient opportunity for learners to achieve their clinical training.

Due to the self-reported nature of RBLs, their utility hinges upon the reliability and accuracy of data entry. Several studies have identified shortcomings with regard to capturing the clinical exposure reported in logbooks periodically updated by medical trainees. ${ }^{6-9}$ Agreement has been reported to be in the range of 30-60\% compared with an a priori defined standard reference of clinical volume that did not include an electronic health record. Simpao et al. ${ }^{10}$ found that electronic health records such as anesthesia information management systems (AIMS) can be queried to generate valid, verifiable case logs of resident involvement. Furthermore, Wanderer et al. ${ }^{11}$ reported that automated forms of comprehensive perioperative documentation (i.e., AIMS) are a feasible means of 
tracking residents' perioperative cases and procedures. While the deficiencies of RLBs may be related to falsified, incomplete, inaccurate, or forgotten cases, electronic logging via AIMS can provide real-time documentation of case exposure. ${ }^{12}$

As anesthesiology residency programs in Canada deliberate on the definition and evaluation of "competence", there is an increasing need to identify reliable, efficient, and accurate methods of assessment. Considering the potential deficiencies with RBLs, we sought to compare their level of agreement with AIMSbased measures of case load during anesthesiology residency. Our data builds on previous studies showing that AIMS data extraction is feasible and representative of residents' perioperative case and procedure volumes. ${ }^{10,11}$ Our primary outcome was total case load with analyses focused on correlation and agreement between these two data sources.

\section{Methods}

Ethics approval for this historical cohort study was received from the Ottawa Health Sciences Network Research Ethics (January 2019, Protocol ID: 20190124$01 \mathrm{H})$. As all data were de-identified, individual consent was not required. This report adhered to the 2015 Standards for Reporting Diagnostic accuracy studies (STARD) guidelines. ${ }^{13,14}$

\section{Setting and data collection}

This study was conducted at The Ottawa Hospital (TOH), a tertiary care academic health sciences network with two inpatient campuses and a free-standing ambulatory surgical centre. The Ottawa Hospital has a catchment area of 1.2 million people and performs $>50,000$ surgical procedures per year. ${ }^{15}$ The Ottawa Hospital is affiliated with the University of Ottawa, which has an anesthesiology residency program accredited by the Royal College of Physicians and Surgeons of Canada. There are eight to ten residents per cohort year and the program is five years in duration. As part of their requirements for residency training, all anesthesiology residents must track their cases using an online RLB (Cissec Corp, Amhersetview, ON; Canada; www.cissec.com/rlb.html) that was developed in 2004 by the Association of Canadian University Departments of Anesthesia. No minimum or maximum number of RLB entries is required; however, all entries are reviewed biannually by the program director and after certain four-week rotations by the module lead (e.g., neuro, thoracic, and vascular anesthesia). Residents were required to make separate entries for each procedure including the surgical specialty, procedure, airway management, any additional monitors used/inserted, the patient's age, American Society of Anesthesiologists' physical status classification, and type of anesthetic. Overall progress is gauged by a review of resident logs compared with their institutional and national average case exposure, which can be found under the "Reports" tab. Resident data from AIMS was extracted from a modified, prospectively collected protocol used by our institution for faculty annual performance reports. ${ }^{16}$ As part of the institution's medicolegal documentation requirements, the resident(s) involved in a given surgical case is identified in the patient's anesthetic record, by either the resident or the staff anesthesiologist, by entering the trainee's name from a dropdown menu. Additional information that is entered includes information regarding surgery (e.g., surgical specialty and procedure) and anesthesia (e.g., airway management, regional anesthetic procedure, and arterial and central venous access). Post hoc extracted data can be individualized by what is determined to be important information.

Data were extracted from RLBs and AIMS into Microsoft Excel (Microsoft, Redmond, WA, USA) and de-identified by our residency program administrator. The reports did not contain patient-specific data and were compliant with the Personal Health Information Protection Act. The administrator aligned each resident's schedule throughout residency with AIMS and RLB data to exclude data logged during periods of time when AIMS was not available. This included offsite elective rotations and rotations at the University of Ottawa (including TOH and other affiliated institutions) outside of the main operating rooms (i.e., obstetrical, pediatric, and cardiac surgery). Data from surgical subspecialties with $<25$ total number cases throughout the five-year residency (typically otorhinolaryngology, ophthalmology, and plastic and maxillofacial surgery) were also excluded to prevent underpowered comparisons. The final data set comprised AIMS and RLB data for seven surgical subspecialties including general, gynecology, neuro, orthopedic, thoracic, urology, and vascular surgery.

\section{Participants}

The study population included all residents who completed the five-year anesthesiology training program between 2011 and $2018(n=27)$. We chose the 2011 cohort as this was the year after AIMS was implemented at TOH. Data for all residents were included and no resident was excluded because of missing values. 
Data analysis

Each resident's total number of cases and number of cases in each surgical subspecialty were calculated from RLB and AIMS data, which were treated as continuous normally distributed variables. Descriptive statistics [mean (standard deviation [SD])] were used to describe these case volumes. All analyses were performed using SAS version 9.4 (SAS Institute, Cary NC, USA), except for the Bland-Altman plots, which were performed using GraphPad Prism (Version 8.0.0; Graphpad Software, San Diego, CA, USA). $P$ values of $<0.05$ were considered to be statistically significant. All analyses were defined $a$ priori; we did not correct for multiple comparisons.

Although the AIMS data can be considered as a medicolegal standard source for documentation, we had no way to verify their accuracy. As we lacked a true "reference standard", we focused strictly on agreement between the AIMS and RLB data. Correlation (the degree to which a change in one variable is correlated with the change in a second variable) is a relatively simple measure related to agreement, but differentiated by the fact that the two values can be highly correlated despite the scales of each measurement or absolute values being quite different. ${ }^{17}$ To determine whether the values derived from each data source were correlated, we calculated Pearson correlation coefficients and $95 \%$ confidence intervals (95\% CI) based on a Fisher transformation. Next, we formally compared agreement using intraclass correlation coefficients (ICCs) and Bland-Altman (BA) plots. Intraclass correlation coefficients measure agreement by considering how the variance in a pair of measures from two or more raters (in this case, RLB and AIMS) explains the overall variance between measures; values close to 1 indicate perfect agreement between raters, while values close to 0 represent total disagreement. ${ }^{18}$ Compared with a Pearson correlation coefficient, an ICC also accounts for bias related to the raters. We used a linear mixed model to calculate two-way mixed effects ICCs $^{19}$ which is a measure of absolute agreement. ${ }^{20}$ Wald $95 \%$ CIs were also calculated for each ICC. Bland-Altman plots (also known as difference plots) more specifically assess the agreement between two instruments used to evaluate the same underlying phenomenon on a continuous scale. To do this, a scatter plot of the average measure on the $\mathrm{X}$-axis $v s$ the difference in the measures on the $\mathrm{Y}$-axis is generated. A plot of perfect agreement would result in a horizontal straight line. ${ }^{18}$ Upper and lower statistical limits of agreement were added to identify outliers and explicit levels of agreement were assigned. These analyses were applied to the total case volumes, as well as to the seven high-volume specialities.
Table 1 Total case volume and as a function of surgical subspecialty ( $n=27$ residents)

\begin{tabular}{lrrr}
\hline Surgical subspecialty & \multicolumn{1}{l}{$\begin{array}{l}\text { RLB } \\
\text { mean (SD) }\end{array}$} & $\begin{array}{l}\text { AIMS } \\
\text { mean (SD) }\end{array}$ & $P$ value \\
\hline General surgery & $150(64)$ & $153(34)$ & 0.76 \\
Gynecology & $55(26)$ & $64(20)$ & 0.04 \\
Neurosurgery & $53(22)$ & $56(13)$ & 0.48 \\
Orthopedic & $178(61)$ & $213(39)$ & $<0.01$ \\
Thoracic & $31(18)$ & $34(10)$ & 0.29 \\
Urology & $65(25)$ & $77(17)$ & 0.03 \\
Vascular & $48(22)$ & $54(14)$ & 0.08 \\
Total cases & $583(191)$ & $649(103)$ & 0.049 \\
\hline
\end{tabular}

AIMS = anesthesia information management system; RLB $=$ resident logbook; SD = standard deviation. $t$ tests were done between RLB and AIMS case logs.

\section{Results}

Overall case exposure

Twenty-seven residents were included in our study. Total mean (SD) case load tracked by RLBs was lower than AIMS [583 (191) vs 649 (103), respectively; $P=0.049$ ] (Table 1; Fig. 1A). Correlation between the two data sources was moderate $(\mathrm{r}=0.50 ; 95 \% \mathrm{CI}, 0.15$ to 0.74$)$ with comparable agreement (ICC, $0.42 ; 95 \%$ CI, 0.34 to 0.59) (Table 2). Bland-Altman plots showed variable agreement with a mean (SD) bias of 66 (166) cases favouring AIMS (Fig. 1B).

\section{Surgical subspecialties}

Secondary outcomes included separate analyses for seven surgical subspecialties including orthopedic, general, gynecology, thoracic, urology, neuro, and vascular surgery. Table 1 depicts descriptive statistics as a function of surgical subspecialty along with measures of correlation and agreement. Overall, the average number of cases recorded in AIMS was greater than that in RLB.

The number of cases logged in AIMS and RLBs were similar for general, neuro, thoracic, and vascular surgery (Table 1 and Fig. 2). For each of these subspecialties, there was moderate correlation ( $\mathrm{r} \geq 0.48)$ and agreement (ICC $\geq$ 0.40, Table 2). Each BA plot showed a statistically insignificant bias.

The remaining surgical subspecialties, including orthopedic, urology, and gynecology, had more logged cases in AIMS relative to RLB by $\sim 20 \%$ (Table 1 and Fig. 3). There was variable correlation such that orthopedic and gynecology logs were moderately correlated $(r \geq 0.55)$ 
A
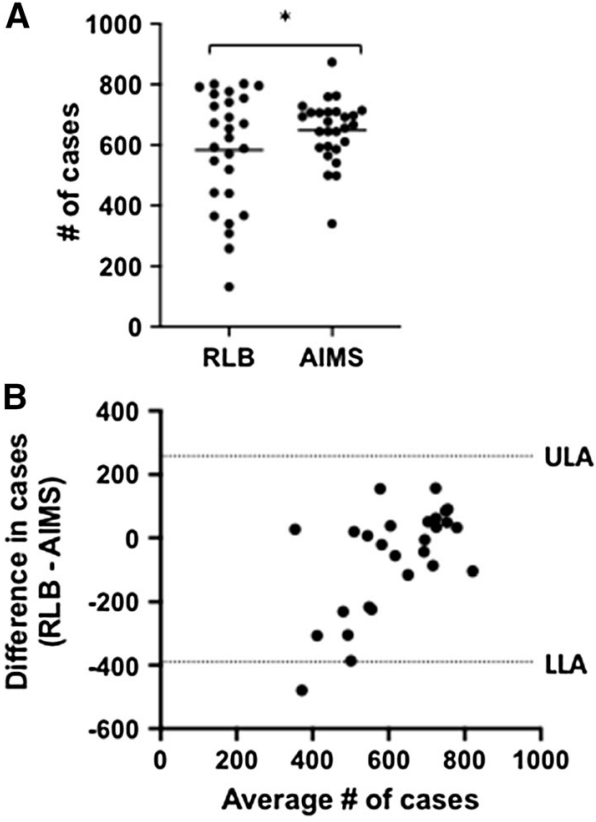

Fig. 1 Total case exposure during anesthesiology residency. Scatter plot (A) and Bland-Altman plot with 95\% limits of agreement (B) of cases logged in a resident-driven logbook (RLB) and an automated logbook (anesthesia information management system [AIMS]). Panel B: Y-axis represents the difference in total cases between RLB and AIMS; $X$-axis represents the mean total number of cases. A value of 0 indicates that an equivalent number of cases were logged in both RLB and AIMS data sources. LLA $=95 \%$ lower limit of agreement; ULA = $95 \%$ upper limit of agreement. ${ }^{*}$ Significantly greater average case exposure reported in AIMS relative to RLB.

with moderate agreement (ICC, 0.53) whereas logs for urology were poorly correlated $(\mathrm{r}=0.25 ; 95 \% \mathrm{CI},-0.14$ to 0.58 ) with poor agreement (ICC, $0.23 ; 95 \% \mathrm{CI}, 0.13$ to $0.74)$.

\section{Discussion}

In this study, we compared the total case load logged through RLB to an automated recording system (based on AIMS) for anesthesiology residents over their five-year residency. Overall, there was a greater number of cases logged in AIMS with only moderate correlation and agreement between the two systems. When parsed by individual subspecialty, there was variability in the differences between AIMS and RLB. In general, neuro, thoracic, and vascular surgery, AIMS and RLB indicated similar case volumes. In contrast, orthopedics, urology, and gynecology had a greater number of logged cases in AIMS.

Logbooks were designed to serve several stakeholders: trainees-verifying that they have adequate exposure to specific types of cases; residency programs-ensuring their trainees have adequate and comparable case loads; and accreditation bodies-ensuring their programs provide sufficient clinical experience for residents to become certified. In light of their utility, logbooks have been widely implemented within the competency-based medical education framework. ${ }^{3,4}$ Our data are consistent with the literature in that there were more cases reported in AIMS than in RLB (Table 1). Furthermore, there appears to be lower inter-resident variability in case load according to AIMS (Fig. 1), which would be expected of a group of residents completing a five-year residency at a single institution. Indeed, the wide variability in self-reported cases among residents in anesthesiology has been shown to be as high as $50 \% .{ }^{21}$ Cumulatively, we believe that these findings support the superiority of an AIMS-based logbook system: this approach allows documentation and tracking of cases in near real-time and circumvents the variable attention to accurate self-reporting. Moreover, there may be the additional benefit of improved resident wellness associated with the reduced administrative responsibility of maintaining a logbook. ${ }^{10-12}$ Automated logbooks may also facilitate the tracking of improved resident performance as clinical experience is accrued (e.g., number of intubation attempts, failed or suboptimal regional/neuraxial procedures).

When case load was divided as a function of surgical subspecialty, there was variable congruence between the AIMS and RLB data. The level of agreement assessed by ICC was similar across subspecialties; however, some subspecialties had a greater number of cases reported in AIMS relative to RLB. Specifically, there was a higher number of cases logged in AIMS for gynecology, orthopedic, and urologic surgery (Table 1). While not assessed in our

study, a considerable portion of these subspecialty cases are done while on-call or during "general anesthesia" rotations, which may contribute to less-accurate manual logging. In contrast, certain subspecialty rotations (e.g., neuro, thoracic, and vascular surgery) have an a priori defined minimum case load requirement coupled with RLB auditing for compliance, which may have led to improved tracking. While regular RLB auditing for all cases would be ideal, this level of surveillance would be resourceintensive. Conversely, the use of an automated logbook may decrease the resources required for such scrutiny. In fact, the financial cost at our institution to extract resident case logs from AIMS over an eight-year period (\$500) was one-quarter of the annual fee incurred to provide all residents access to RLBs.

While there are advantages to case-logging via AIMS, we would draw attention to the following caveats. First, not all university departments and hospitals are equipped with AIMS technology; in our study, this was the case in cardiac, obstetric, and pediatric anesthesia rotations. We anticipate that as AIMS uptake increases across Canada 
Fig. 2 Bland-Altman plot depicting agreement and $95 \%$ upper and lower limits of agreement of cases logged in resident-driven logbook (RLB) and an automated logbook (anesthesia information management system [AIMS]) for general surgery, neurosurgery, thoracic, and vascular surgery procedures. Yaxis represents the difference in cases between RLB and AIMS; $\mathrm{X}$-axis is the mean number of cases for that surgical subspecialty. A value of 0 indicates that an equivalent number of cases were logged in both data sources. LLA $=95 \%$ lower limit of agreement; ULA $=95 \%$ upper limit of agreement.
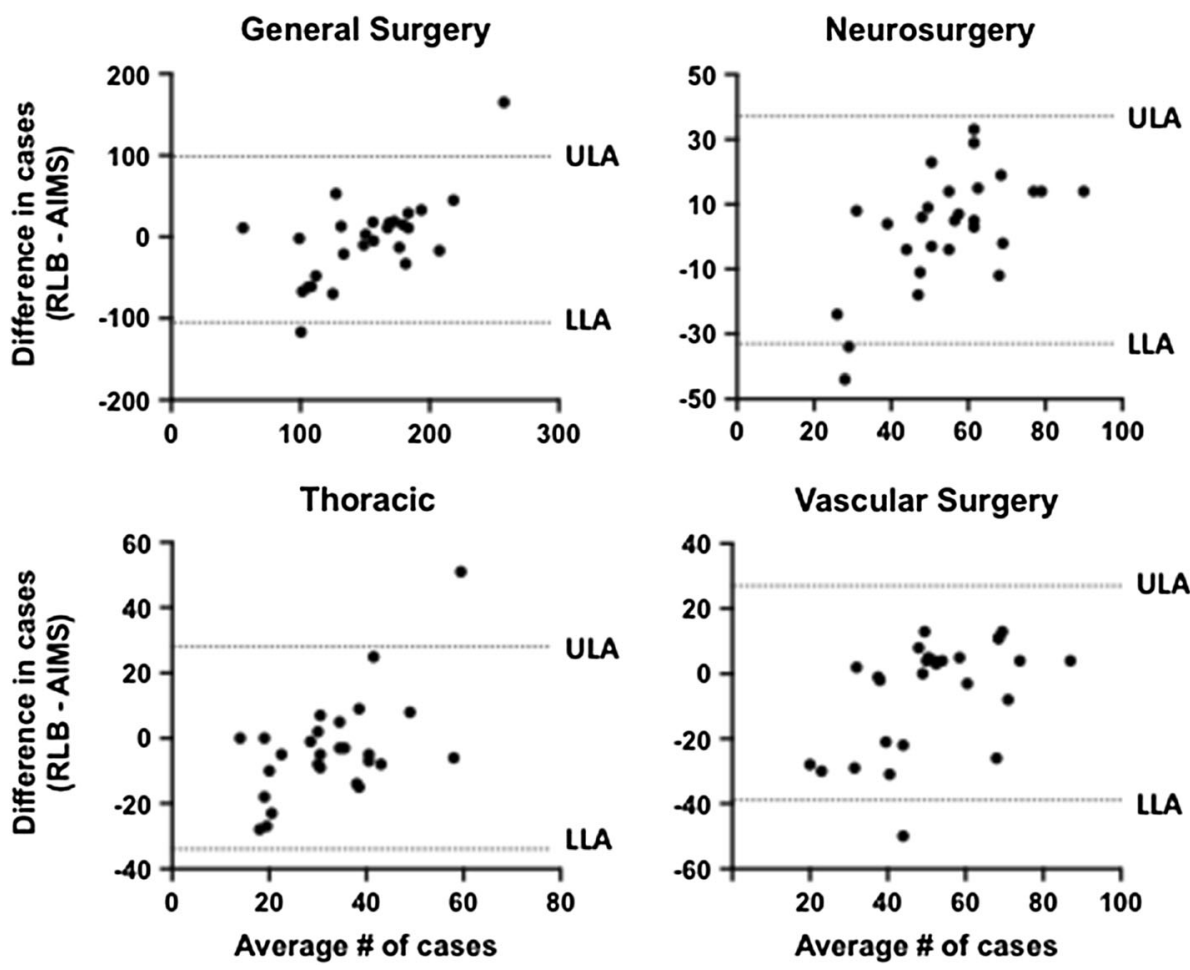

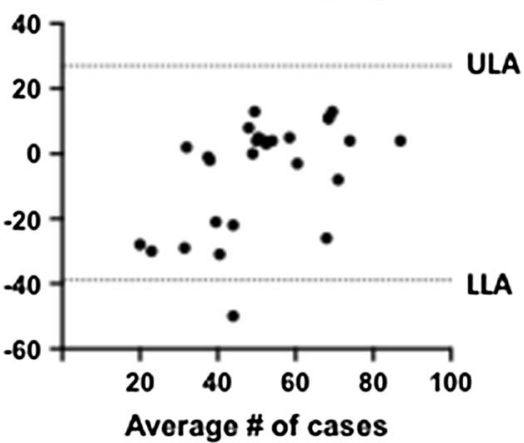

Table 2 Correlation and agreement between RLB and AIMS for total cases and as a function of surgical subspecialty ( $n=27$ residents)

\begin{tabular}{lll}
\hline Surgical subspecialty & Pearson $\mathrm{r}(95 \% \mathrm{CI})$ & ICC $(95 \% \mathrm{CI})$ \\
\hline General surgery & $0.59(0.27$ to 0.79$)$ & $0.48(0.42$ to 0.61$)$ \\
Gynecology & $0.55(0.22$ to 0.77$)$ & $0.53(0.48$ to 0.63$)$ \\
Neurosurgery & $0.60(0.29$ to 0.80$)$ & $0.52(0.46$ to 0.62$)$ \\
Orthopedic & $0.59(0.27$ to 0.79$)$ & $0.53(0.48$ to 0.63$)$ \\
Thoracic & $0.48(0.09$ to 0.71$)$ & $0.40(0.30$ to 0.59$)$ \\
Urology & $0.25(-0.14$ to 0.58$)$ & $0.23(0.13$ to 0.74$)$ \\
Vascular & $0.62(0.32$ to 0.81$)$ & $0.57(0.53$ to 0.65$)$ \\
Total cases & $0.50(0.15$ to 0.74$)$ & $0.42(0.34$ to 0.59$)$
\end{tabular}

AIMS = anesthesia information management system; $\mathrm{CI}=$ confidence interval; ICC $=$ intraclass correlation coefficient (using two-way mixed effects ICC) $;^{19}$ RLB $=$ resident logbook.

this will eventually cease to be a limiting factor. Second, use of a common information platform by Canadian residency programs (as provided by the RLB) facilitates comparison of data acquired at each institution. For example, using the "Reports" section in the RLB, residents can generate their real-time reports and compare them with the program and national averages. With AIMS, a common information platform could be achieved using a standardized set of elements for data entry, a centralized collation of data, and the generation of reports at fixed intervals that accurately captures the individual, program, and national anesthesia resident experience at Canadian institutions.

There are limitations to our study that should be considered. First, because of variable AIMS technology availability, our data sample was limited to surgeries performed in operating rooms at the main teaching hospital of our anesthesia residency program. Therefore, logging of cases from other teaching hospitals (including all cardiac surgery, obstetric, pediatric cases), community hospitals, and elective rotations was not possible. A second limitation is that we had no gold reference standard to ensure (or ascertain) accuracy of case-logging. Other studies have attempted to identify a reference standard (e.g., records kept by senior physicians) but these may not represent a true gold standard. ${ }^{6-9}$ Indeed, it is challenging to identify a reliable gold standard. Resident logging habits were variable such that some residents logged cases weekly, while others would have no entries for several months before submitting a large number of cases, as noted by others. $^{22}$ This calls into question the accuracy and reliability of RLB data entry, particularly when there are considerable time lags between cases and logging. Similarly, we have no way of assessing the instances when residents were accidently omitted from the AIMS record. This could be addressed, in part, by making learner involvement a mandatory field of data entry (currently, there is no prompt to enter any learner involved in the case). Third, our sample size was limited to one of 

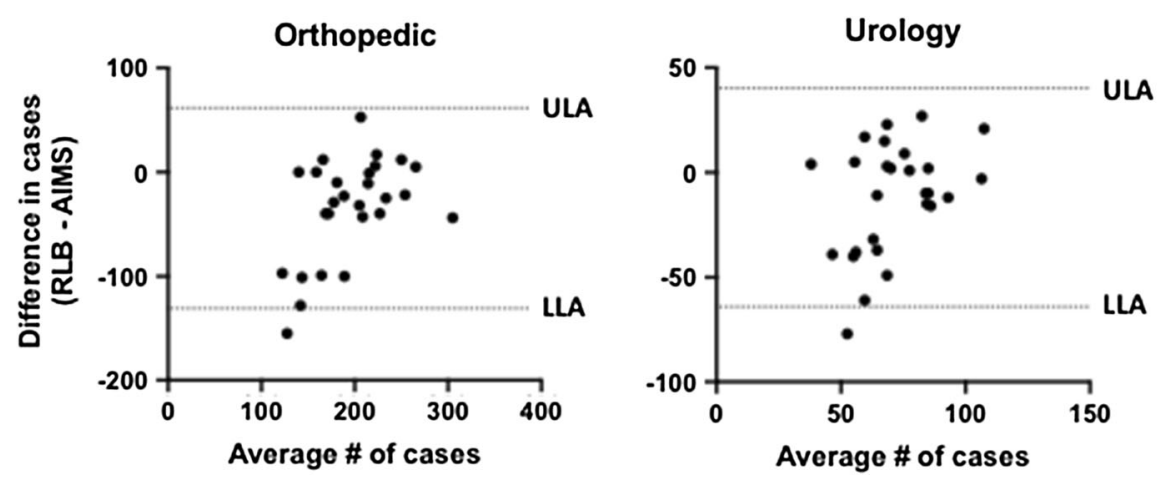

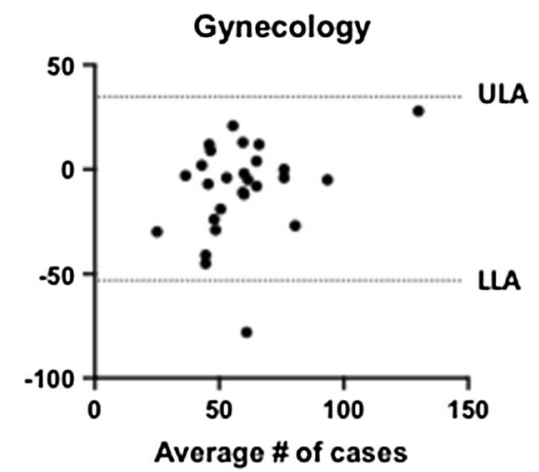

Fig. 3 Bland-Altman plots depicting agreement and 95\% upper and lower limits of agreement of case exposure logs comparing a residentdriven and an automated logbook for orthopedic, gynecology, and urology procedures. The data sources are the resident-driven logbook (RLB) compared with an automated logbook (anesthesia information management system [AIMS]). Y-axis represents the difference in cases between RLB and AIMS; X-axis is the mean number of cases for that surgical subspecialty. A value of 0 indicates that an equivalent number of cases were logged in both data sources. LLA $=95 \%$ lower limit of agreement; ULA $=95 \%$ upper limit of agreement. convenience: the cohort of residents who completed their five-year residency while contemporaneously using RLB and AIMS. We acknowledge that our observations must be tempered by the small number of residents involved in the study. Finally, while we defined our comparisons a priori, we did not correct for multiple comparisons, which slightly increases the risk of a type- 1 error.

In conclusion, when comparing cases logged using RLBs $v s$ AIMS during anesthesia residency, we found that the volumes were higher and showed lower variability with AIMS. As AIMS data represent a medico-legal standard, are routinely collected, and place minimal administrative burden on residents, we believe that automated log booking is superior. Future efforts will be required to establish standards for AIMS-based case logs, integrate automated logbooks across hospital networks, and ultimately establish a common information platform that can be used by anesthesia residency programs across Canada.

Author contributions Daniel Dubois (principal investigator and project leader) was involved in project design and management, manuscript writing, and dissemination. Ryan McGinn and Alexander Lingley (co-investigators) were involved in data analysis, manuscript writing, and dissemination. Meghan McConnell (co-investigator) was involved in project design and management, data analysis, manuscript writing, and dissemination. Chris Pysyk and Gregory L. Bryson (coinvestigators) were involved in project design and manuscript writing.

\section{Disclosures None.}

Funding statement Department of Innovation in Medical Education, University of Ottawa.

Editorial responsibility This submission was handled by Dr. Steven Backman, Associate Editor, Canadian Journal of Anesthesia.

\section{References}

1. Fraser AB, Stodel EJ, Chaput AJ. Curriculum reform for residency training: competence, change, and opportunities for leadership. Can J Anesth 2016; 63: 875-84.

2. Holmboe ES, Sherbino J, Long DM, Swing SR, Frank JR. The role of assessment in competency-based medical education. Med Teach 2010; 32: 676-82.

3. Maudsley RF, Dagg P, Wilson J, et al. Competence by Design: Reshaping Canadian Medical Education. Royal College of Physicians and Surgeons of Canada; 2014. Available from URL: http://www.royalcollege.ca/rcsite/documents/educationalstrategy-accreditation/royal-college-competency-by-designebook-e.pdf (accessed May 2020).

4. Royal College of Physicians and Surgeons of Canada. Standards of Accreditation for Residency Programs in Anesthesiology. Office of Specialty Education Editorial revision \#2. Ottawa, ON, Canada. January 2019. Available from URL: http://www. royalcollege.ca/rcsite/documents/ibd/anesthesiology-sa-e.pdf (accessed May 2020).

5. Schuttpelz-Brauns $K$, Narciss E, Schneyinck C, et al. Twelve tips for successfully implementing logbooks in clinical training. Med Teach 2016; 38: 564-9.

6. Witt A, Iglesias S, Ashbury T. Evaluation of Canadian family practice anesthesia training programs: can the resident logbook help? Can J Anesth 2012; 59: 968-73.

7. Chou S, Lockyer J, Cole G, McLaughlin K. Assessing postgraduate trainees in Canada: are we achieving diversity in methods? Med Teach 2009; 31: e58-63.

8. Luke C, Kadzombe E, Armstrong A, Gorman D, Horner J. An evaluation of a logbook for trainees in accident and emergency medicine in the United Kingdom. Arch Emerg Med 1991; 8: 130-4.

9. Raghoebar-Krieger HM, Sleijfer D, Bender W, Stewart RE, Popping $R$. The reliability of logbook data of medical students: an estimation of interobserver agreement, sensitivity and specificity. Med Educ 2001; 35: 624-31.

10. Simpao AF, Pruitt EY, Cook-Sather SD, Gurnaney HG, Rehman $M A$. The reliability of manual reporting of clinical events in an anesthesia information management system (AIMS). J Clin Monit Comput 2012; 26: 437-9. 
11. Wanderer JP, Charnin J, Driscoll WD, Bailin MT, Baker K. Decision support using anesthesia information management system records and accreditation council for graduate medical education case logs for resident operating room assignments. Anesth Analg 2013; 117: 494-9.

12. Galvez JA, Rothman BS, Doyle CA, Morgan S, Simpao AF, Rehman MA. A narrative review of meaningful use and anesthesia information management systems. Anesth Analg 2015; 121: 693-706.

13. Cohen JF, Korevaar DA, Altman DG, et al. STARD 2015 guidelines for reporting diagnostic accuracy studies: explanation and elaboration. BMJ Open 2016; DOI: https://doi.org/10.1136/ bmjopen-2016-012799.

14. Bossuyt PM, Cohen JF, Gatsonis CA, Korevaar DA, STARD Group S. STARD 2015: updated reporting guidelines for all diagnostic accuracy studies. Ann Transl Med 2016; DOI: https:// doi.org/10.3978/j.issn.2305-5839.2016.02.06.

15. The Ottawa Hospital. The Ottawa Hospital Annual Report 20182019: Fast Facts. Available from URL: https://www. ottawahospital.on.ca/annualreport/fast-facts_en.html (accessed May 2020).

16. Wheeler K, Baxter A, Boet S, Pysyk C, Bryson GL. Performance feedback in anesthesia: a post-implementation survey. Can J Anesth 2017; 64: 681-2.
17. Bartko JJ. The intraclass correlation coefficient as a measure of reliability. Psychol Rep 1966; 19: 3-11.

18. Bland JM, Altman DG. Statistical methods for assessing agreement between two methods of clinical measurement. Lancet 1986; 1: 307-10.

19. Shrout PE, Fleiss JL. Intraclass correlations: uses in assessing rater reliability. Psychol Bull 1979; 86: 420-8.

20. Koo TK, Li MY. A Guideline of selecting and reporting intraclass correlation coefficients for reliability research. J Chiropr Med 2016; 15: 155-63.

21. Yamamoto S, Tanaka P, Madsen MV, Macario A. Analysis of resident case logs in an anesthesiology residency program. A A Case Rep 2016; 6: 257-62.

22. Cadish LA, Fung V, Lane FL, Campbell EG. Surgical case logging habits and attitudes: a multispecialty survey of residents. J Surg Educ 2016; 73: 474-81.

Publisher's Note Springer Nature remains neutral with regard to jurisdictional claims in published maps and institutional affiliations. 